DOI: $10.12731 /$ wsd-2018-5-46-61

УДК 616.151.5-073.96

\title{
НАЧАЛЬНЫЙ ЭТАП \\ ТЕСТИРОВАНИЯ РАЗРАБАТЫВАЕМОГО АППАРАТА ДЛЯ ОПРЕДЕЛЕНИЯ МЕЖДУНАРОДНОГО НОРМАЛИЗОВАННОГО ОТНОШЕНИЯ
}

Аксотина Н.В., Шульман В.А., Зограф Ф.Г., Мордовский В.С., Маринушкин П.С., Гавриленко М.И., Кусаев В.В., Кононов Е.Н., Петров С.С., Семенов Э.В., Князева И.С.

Протромбиновое время (ПТВ) - это лабораторный показатель, позволяющий оценить внешний путь свертывания крови (активность факторов I, II, V, VII и X). Для стандартизачии результатов теста ПТВ введен показатель МНО. МНО = (ПТВ паџиента/ПТВ 100\%)МИЧ. Набирает популярность контроль МНО с помощьью портативных коагулометров. На российском рынке представлены: CoaguChek XS, qLabs Electrometer. Главная проблема - их высокая стоимость и дороговизна в эксплуатации. Российские ученье давно задались вопросом импортозамещения. Однако, в литературе нет информаџии о тестировании разработок. При разработке экспериментальной модели для измерения МНО и ПТВ, мы руководствовались результатами литературного поиска по наукометрическим базам данных Elibrary, Scopus, PubMed u WoS с учетом предполагаемой стоимости разрабатываемой модели.

Цель исследования. Провести начальный этап тестирования экспериментальной модели с сопоставлением полученных результатов с данными сертифицированной лаборатории.

Материал и методы. Обследовано 70 пациентов (26 мужчин и 44 женщины), принимающих «Варфарин». Материал для измерения экспериментальной моделью - капиллярная кровь. В качестве тест-системы применяли тест-полоску qLabs ${ }^{\circledR}$ PT-INR Test Strip.

Результаты. Разница ПТВ тестируемой модели и результатов сертифииированной лаборатории \pm 1-2 секунды. ПТВ Протромбин-калибратора 13 сек. Протромбиновое отночение Протромбин-калибратора 1,0. Пациент №1. ПТВ на тестируемом аппарате 34 сек. МИЧ тробопластина на тест-полоске 1,0. МНО $=(34 \text { сек } / 13 \text { сек } \times 1,0)^{1,0}=2$,62. Результат сер- 
тифицированной лаборатории: ПТВ 36 сек, МНО 2,86. Пачиент №2. ПТВ на тестируемом аппарате 31 сек. $\mathrm{MHO}=(31 \text { сек } / 13 \text { сек } \times 1,0)^{1,0}=2,38$. Результаты сертифицированной лаборатории: ПТВ 32 сек, МНО 2,48.

Вывод. Согласно представленным результатам, полученным в ходе тестирования разработанной портативной модели у пациентов, находяшихся на варфаринотерапии, получены сопоставимые результаты ПТВ и МНО сертифицированной лабораторией.

Ключевые слова: международное нормализованное отночение; протромбиновое время; коагулометр; Варфарин.

\title{
THE INITIAL STAGE OF TESTING THE DEVELOPED APPARATUS TO DETERMINE THE INTERNATIONAL NORMALIZED RATIO
}

\author{
Aksyutina N.V., Shulman V.A., Zograf F.G. Mordovskiy V.S., \\ Minushkin P.S., Gavrilenko M.I., Kusaev V.V., Kononov E.N., \\ Petrov S.S., Semenov E.V., Knyazeva I.S.
}

Prothrombin time (PTT) is a laboratory indicator that allows to evaluate the external pathway of blood coagulation (activity of factors I, II, V, VII and X). An INR indicator was introduced to standardize the results of the PTV test. INR = (patient's PTT/PTT norm) ISI. The control of the INR is gaining popularity with the help of portable coagulometers. On the russian market are CoaguChek XS, qLabs Electrometer. The main problem is their high cost and high cost of operation. Russian scientists have long wondered about import substitution. However, in the literature there is no information on testing development. When developing an experimental model for measuring INR and PTV, we were guided by the results of the literary search in the scientometric databases Elibrary, Scopus, PubMed and WoS, taking into account the estimated cost of the developed model.

Background. To conduct the initial stage of testing an experimental model with a comparison of the results obtained with the data of a certified laboratory.

Materials and methods. We examined 70 patients (26 men and 44 women) taking «Warfarin». The material for measuring the experimental model was capillary blood. We used the qLabs ${ }^{\circledR}$ PT-INR Test Strip as a test system.

Results. We obtained the Difference of the PTT of the tested model and the results of the certified laboratory $\pm 1-2$ seconds. PTT prothrombin calibrator was 13 sec. Prothrombin ratio prothrombin calibrator was 1.0. Patient №1. 
PTT on the tested device 34 seconds. ISI troboplastin on test strip was 1.0. INR = $(34 \mathrm{sec} / 13 \mathrm{sec} \times 1.0)^{1.0}=2.62$. The result of a certified laboratory: PTT $36 \mathrm{sec}$, INA 2.86. Patient №2. PTT on the tested device $31 \mathrm{sec}$. INR $=(31 \mathrm{sec} / 13 \mathrm{sec} \times$ 1.0) $)^{1.0}=2.38$. The results of the certified laboratory: PTT $32 \mathrm{sec}$, INR 2.48 .

Conclusions. According to the presented results obtained during testing of the developed portable model in patients on warfarin therapy, comparable results of PTT and INA were obtained with a certified laboratory.

Keywords: international normalized ratio; prothrombin time; coagulometer; Warfarin.

Протромбиновое время (ПТВ) - это лабораторный показатель, отображающий время образования сгустка после добавления в плазму тканевого фактора (тромбопластина). Протромбиновый индекс (ПТИ) и международное нормализованное отношение (МНО) - производные ПТВ $[1,2]$. Данные показатели позволяют оценить внешний путь свертывания крови и систему гемостаза в целом (активность факторов свертывания I, II, V, VII и X). Это является необходимым при терапии «Варфарином», в случае нарушения печеночной функции (синтеза факторов коагуляции), для оценки степени насыщения витамином К. Нормальный показатель ПТВ $11-16$ сек [1-3].

Скорость коагуляции крови по внешнему пути напрямую зависит от концентрации VII фактора свертывания крови. VII фактор - белок с короткой продолжительностью жизни, для его синтеза необходим витамин К. Удлинение ПТВ может быть при дефиците витамина К, возникающего при применении «Варфарина» (желательный клинический эффект), при синдроме мальабсорбции, при недостаточной бактериальной колонизации кишечника (например, у новорожденных). Кроме того, недостаточная концентрация VII фактора может наблюдаться при заболеваниях печени (нарушен синтез) или при повышенном потреблении VII фактора (ДВС-синдроме), вследствие чего происходит удлинение ПТВ $[1,4]$. При определении ПТВ в сертифицированной лаборатории чаще всего используется плазма крови. У новорожденных для анализа берется цельная кровь. Результат ПТВ у здоровых людей варьирует в зависимости от типа реагента - тканевого фактора (тромбопластина). Чтобы стандартизировать результаты теста ПТВ в клиническую практику был введен показатель МНО. Производители реагента (тромбопластина) для теста ПТВ указывают международный индекс чувствительности (МИЧ). МИЧ показывает активность тканевого фактора в данной произведенной партии реаген- 
та в сравнении со стандартизованным образцом. Значения МИЧ обычно между 1,0 и 2,0 [1]. МНО рассчитывается как отношение ПТВ пациента к нормальному ПТВ (100\%), возведенное в значение МИЧ использованного при тесте реагента [1-3]:

$$
\mathrm{MHO}=(\text { ПТВ пациента/ПТВ 100\%) })^{\text {мич }}
$$

МНО в норме у здорового человека от 0,8 до 1,3. При приеме «Варфарина» целевой уровень МНО 2,0-3,0, хотя в отдельных случаях, например, при наличии искусственного механического клапана сердца, в предоперационный период может потребоваться более высокий целевой уровень МНО. При МНО выше 5,0 наблюдается высокий риск кровотечения, при МНО ниже 0,5 - высокий риск тромбообразования [5,6]. В настоящее время, помимо лабораторного контроля, набирает популярность мониторирование МНО с помощью портативных коагулометров в домашних условиях с высокой точностью результатов [7-12].

На сегодняшний день на российском рынке представлены две модели автоматических портативных коагулометров с возможностью для домашнего использования: Коагучек Икс Эс (CoaguChek XS), КуЛабс Электрометр (qLabs Electrometer). Характеристики приборов схожие, представлены в таблице №1, их использование не вызывает затруднения. Главной проблемой портативных коагулометров остается их высокая стоимость и дороговизна в эксплуатации, не доступная большинству пациентов [13-16]. Во многих малонаселенных местностях, далеко расположенных от сертифицированных лабораторий, остается проблема контроля МНО. Поэтому появление отечественного аналога в более низкой ценовой категории позволит частично решить данную проблему.

Российские ученые давно задались вопросом импортозамещения. Однако, в литературе нет информации о тестировании разработок, сопоставлении данных с результатами сертифицированной лаборатории. Несмотря на имеющиеся разработки, отечественный коагулометр до настоящего времени не представлен на российском рынке.

При разработке экспериментальной модели, предназначенной для измерения МНО и ПТВ в домашних условиях, мы руководствовались результатами литературного поиска по наукометрическим базам данных Elibrary, Scopus, PubMed и WoS с учетом предполагаемой стоимости разрабатываемой модели.

Цель исследования: Провести начальный этап тестирования экспериментальной модели с сопоставлением полученных результатов с данными сертифицированной лаборатории. 


\section{Материал и методы}

Нами проведено исследование 70 пациентов (26 мужчин и 44 женщины), принимающих «Варфарин». Пациенты находились на стационарном лечении в КГБУЗ «КМКБ № 20 им. И.С. Берзона». Медиана возраста 59,00 лет $[47,00-65,00]$. В соответствии с Хельсинской декларацией от 2013 года, для проведения исследования было получено разрешение Локального Этического комитета при КрасГМУ им. проф. В.Ф. Войно-Ясенецкого (Выписка из протокола заседания №84/2018 от 6 июня 2018 г.). Материалом для измерения экспериментальной модели являлась капиллярная кровь, взятая обученным персоналом с соблюдением правил асептики и антисептики. Каждый участник исследования подписывал информированное согласие. В данном исследовании в качестве тест-системы применяли тест-полоску qLabs ${ }^{\circledR}$ PT-INR Test Strip. Параллельно проводился забор крови в вакутейнер с цитратом натрия для последующего определения ПТВ и МНО в сертифицированной лаборатории гемостаза в течение часа. При включении в исследование, пациентов информировали о современных возможностях измерения МНО в домашних условиях при помощи портативных коагулометров, предоставляли сравнительную характеристику портативных коагулометров (табл. 1).

Таблицуа 1.

Сравнительная характеристика портативных коагулометров, представленных на российском рынке

\begin{tabular}{|c|c|c|}
\hline Характеристика & $\begin{array}{l}\text { Коагучек Икс Эс } \\
\text { (CoaguChek XS) }\end{array}$ & $\begin{array}{l}\text { КуЛабс Электрометр } \\
\text { (qLabs Electrometer) }\end{array}$ \\
\hline $\begin{array}{c}\text { Страна производи- } \\
\text { тель }\end{array}$ & Германия & Китай \\
\hline Тип образца & $\begin{array}{c}\text { свежая цельная капиллярная } \\
\text { кровь или цельная венозная кровь }\end{array}$ & $\begin{array}{c}\text { свежая цельная } \\
\text { капиллярная кровь }\end{array}$ \\
\hline Погрешность & $\begin{array}{c}\text { максимальная погрешность } \\
\text { при анализе капиллярной крови } \\
\text { составляет } \pm 4,5 \%, \text { а при анализе } \\
\text { венозной крови } \pm 3,5 \% .\end{array}$ & $<5 \%$ \\
\hline $\begin{array}{l}\text { Диапазон } \\
\text { измерений }\end{array}$ & $0,8-8$ & $0,5-7,5$ \\
\hline Метод измерения & Электрохимический & Электрохимический \\
\hline $\begin{array}{l}\text { Связь с персональ- } \\
\text { ным компьютером }\end{array}$ & $\begin{array}{l}\text { С помощью устройства } \\
\text { Coaguchek XS Connect } \\
\text { (приобретается отдельно) }\end{array}$ & $\begin{array}{c}\text { C помощью USB-кабе- } \\
\text { ля (входит в комплект } \\
\text { поставки) }\end{array}$ \\
\hline
\end{tabular}


Окончание табл. 1.

\begin{tabular}{|c|c|c|}
\hline Вес & 127 г. (без элементов питания) & $\begin{array}{c}126 \text { г. (без элементов } \\
\text { питания) }\end{array}$ \\
\hline $\begin{array}{c}\text { Цена на 12.11.2018 } \\
\text { год по результатам } \\
\text { самостоятельного } \\
\text { анализа цен } \\
\text { поставщиков }\end{array}$ & От 35500 руб. & От 19490 руб. \\
\hline $\begin{array}{c}\text { Цена на тест- } \\
\text { полоски на } \\
12.11 .2018 \text { год } \\
(24 \text { шт.) по результа- } \\
\text { там самостоятельно- } \\
\text { го анализа цен } \\
\text { поставщиков }\end{array}$ & От 7100 руб. (1 шт. $-295,83$ & От 5300 руб. \\
(1шт. $-220,8$ руб.)
\end{tabular}

\section{Результаты и обсуждение}

До тестирования экспериментальной модели мы провели анкетирование пациентов. Результаты анкетирования: портативный коагулометр имеется у 7 пациентов (10,00\%); коагулометр CoaguChek XS - у 3 человек, коагулометр Micropoint qLabs ElectroMeter - у 4 человек. Из них продолжают регулярный контроль МНО в домашних условиях 4 пациента, остальные отказались в связи с финансовыми трудностями в приобретении тест-полосок. Последующие вопросы анкеты позволили выяснять причины, по которым 63 пациента (90,00\%) не приобретают портативный коагулометр для измерения МНО. Из них 19 пациентов (30,16\%) регулярно контролирует МНО в районной поликлинике. 31 человек $(49,21 \%)-$ контролируют МНО в районной поликлинике не регулярно, они хотели бы приобрести коагулометр, но не имеют финансовой возможности, 13 пациентов $(20,63 \%)$ не понимают необходимость и важность постоянного контроля МНО (рис. 1).

Также, следует отметить, что 14 (20,00\%) анкетированных не получали ранее информации о возможности домашнего контроля МНО, 4 (5,71\%) из них в подгруппе регулярно контролирующих МНО в районной поликлинике, 5 (7,14\%) - из контролирующих МНО в районной поликлинике не регулярно, желающих приобрести коагулометр, но не имеющих финансовой возможности, 5 (7,14\%) пациентов - в подгруппе не понимающих необходимость и важность постоянного контроля МНО. Таким образом, мы подтвердили необходимость в разработке российского коагулометра. 


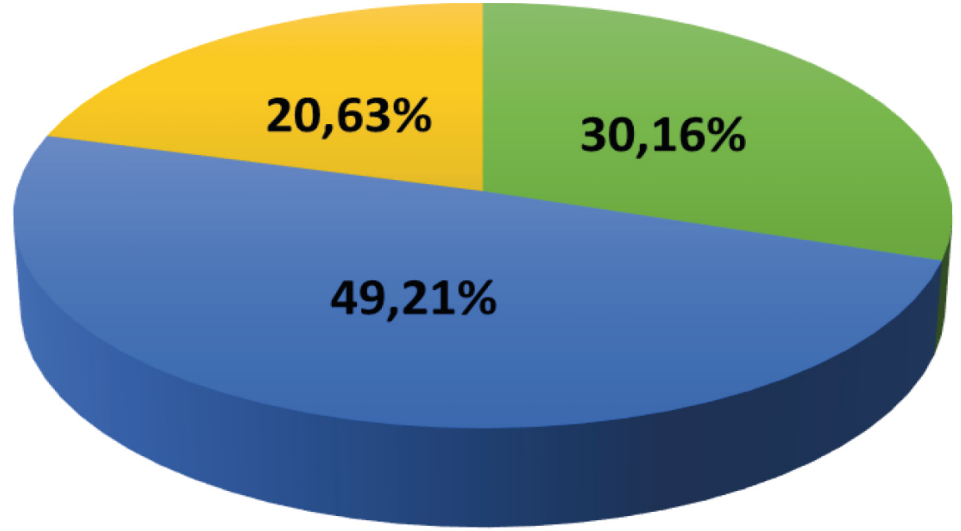

— Регулярно контролируют МНО в районной поликлинике

- Материальные трудности

\section{- Не понимают важность и необходимость контроля МНО}

Рис. 1. Распределения причин не приобретения портативного коагулометра среди пациентов, нуждающихся в регулярном контроле МНО

Результаты тестирования экспериментальной модели показали, что разница полученных результатов при сопоставлении с результатами сертифицированной лаборатории показателя ПТВ оказалась незначительной, она составляла $\pm 1-2$ сек.

Приведем примеры сопоставления результатов.

Первое измерение тестируемым макетом сделано с использованием Протромбин-калибратора для определения нормального показателя ПТВ с последующим внесением его в формулу расчета МНО. При нанесении капли Протромбин-калибратора в стандартном разведении на тест-систему тестируемого аппарата определено протромбиновое время Протромбин-калибратора (ПТВпк), которое составило 13 сек. Протромбиновое отношение Протромбин-калибратора (ПОпк) согласно прилагаемому паспорту $=1,0$.

МНО рассчитывался по формуле:

$$
\text { MHO }=(\text { ПТВ пациента } / \text { ПТВпк } \times \text { ПОпк })^{\text {мич }}
$$

Пациент №1. Результат ПТВ на тестируемом аппарате 34 сек. Учитывая, что МИЧ ТП на тест-полоске 1,0. Соответственно: 


$$
\mathrm{MHO}=(34 \text { сек } / 13 \text { сек } \times 1,0)^{1,0}=2,62 .
$$

Согласно полученному результату анализа в сертифицированной лаборатории: ПТВ 36 сек, МНО 2,86.

Пациент №2. Результат ПТВ на тестируемом аппарате 31 сек.

$$
\mathrm{MHO}=(31 \text { сек } / 13 \text { сек } \times 1,0)^{1,0}=2,38
$$

Результаты сертифицированной лаборатории: ПТВ 32 сек, МНО 2,48.

\section{Вывод}

Согласно представленным результатам, полученным в ходе тестирования разработанной портативной модели у пациентов, находящихся на варфаринотерапии, получены сопоставимые результаты ПТВ и МНО с сертифицированной лабораторией.

Внесение полученных данных в формулу расчета, при знании ПТВпк, ПОпк, учитывая МИЧ используемого при исследовании тромбопластина, позволяет определить показатель МНО. Однако, эти результаты не могут быть использованы в клинической практике, так как являются чисто экспериментальными, необходимыми для подтверждения в правильности выбора электрохимического метода.

Потребность в доступном отечественном портативном коагулометре для домашнего использования не вызывает сомнения, так как на сегодняшний день остается актуальной проблема регулярного контроля МНО, особенно среди пациентов маломобильных и удаленно живущих от крупных населенных пунктов. Остается высокая частота встречаемости осложнений от приема антагонистов витамина К, таких как кровотечения и недостаточный терапевтический эффект от приема вследствие ненадлежащего контроля МНО [1-3]. Использование пациентами автоматических портативных коагулометров позволит улучшить ситуацию по контролю MHO, а использование портативного коагулометра посредством телемедицины и/или специальных приложений на телефоне позволит улучшить контроль МНО и уменьшить нагрузку на медицинскую сеть, тем самым снижая расходы на здравоохранение $[8,9,15,16]$.

\section{Благодарность}

Исследование выполнено при поддержке краевого государственного автономного учреждения «Красноярский краевой фонд поддержки научной и научно-технической деятельности» в рамках реализации проекта: «Разработка портативного коагулометра для контроля международного нормализованного отношения». 


\section{Список литературы}

1. Скрининговые тесты плазменного гемостаза протромбиновое время, АЧТВ, тромбиновое время, фибриноген / Берковский А.Л., Ергеева Е.В., Простакова Т.М., Мелкумян А.Л., Суворов А.В. М., 2016. 70 с.

2. Титаева Е.В., Добровольский А.Б. Возможные источники ошибок при определении МНО и пути их решения // Атеротромбоз. 2015. № 2. С. 107 114. doi:10.21518/2307-1109-2015-2-107-114

3. Guidelines for thromboplastin and plasma used to control oral anticoagulant therapy // WHO Expert Committee on Biological Standartization. Report 48. World Health Organization. 1999, pp. 64-69. (WHO technical report series; 889)

4. Effects of warfarin on biological processes other than haemostasis: A review / Popov A., Mirkov I., Ninkov M., Mileusnic D., Demenesku J., Subota V., Kataranovski D., Kataranovski M. // Food and Chemical Toxicology. 2018. vol. 113, pp. 19-32. doi:10.1016/j.fct.2018.01.019

5. INR Control of Patients with Mechanical Heart Valve on Long-Term Warfarin Therapy / Tan C.S.Y., Fong A.Y.Y., Jong Y.H., Ong T.K. // Global Heart. 2018. vol. 13, № 4, pp. 241-244. doi:10.1016/j.gheart.2018.08.003

6. Warfarin treatment quality and prognosis in patients with mechanical heart valve prosthesis / Grzymala-Lubanski B., Svensson P.J., Renlund H., Jeppsson A., Själander A. // Heart. 2016. vol. 103, № 3, pp. 198-203. doi:10.1136/heartjnl-2016-309585

7. Home Management of Warfarin Treatment Through a Real-Time Supervised Telemedicine Solution: A Randomized Controlled Trial / Brasen C.L., Madsen J.S., Parkner T., Brandslund I. // Telemedicine and e-Health. 2018. doi:10.1089/ tmj. 2017.0260

8. User Evaluation of a Smartphone Application for Anticoagulation Therapy / Smaradottir B., Martinez S., Borycki E., Loudon G., Kushniruk A., Jortveit J., Fensli R. // Studies in Health Technology and Informatics. 2018. vol. 247, pp. 466-470. doi:10.3233/978-1-61499-852-5-466

9. 2016 ESC Guidelines for the management of atrial fibrillation developed in collaboration with EACTS / Kirchhof P., Benussi S., Kotecha D., Ahlsson A., Atar D., Casadei B., Castella M., Diener H.C., Heidbuchel H., Hendriks J., Hindricks G., Manolis A.S., Oldgren J., Popescu B.A., Schotten U., Van Putte B., Vardas P. // European Heart Journal. 2016. vol. 37, № 38, pp. 2893-2962. doi: 10.1093/eurheartj/ehw210

10. Warfarin control in patients transitioning to warfarin after non-vitamin $\mathrm{K}$ oral anticoagulant (NOAC) therapy / Bernaitis N., Badrick T., Davey A.K., Crilly J., Anoopkumar-Dukie S. // Journal of Thrombosis and Thrombolysis. 2018. vol. 46, № 4, pp. 461-465. doi: 10.1007/s11239-018-1719-x 
11. Skaistis J., Tagami T. Risk of Fatal Bleeding in Episodes of Major Bleeding with New Oral Anticoagulants and Vitamin K Antagonists: A Systematic Review and Meta-Analysis // PLoS One. 2015. vol. 10, № 9. P. e0137444. doi: 10.1371/ journal.pone. 0137444

12. Banfi G., Del Fabbro M. (2008). Biological variation in tests of hemostasis // Seminars in Thrombosis and Hemostasis. 2008. vol. 34, № 7, pp. 635-641. doi:10.1055/s-0028-1104541

13. Chen Q., Shou W., Wu W., Guo Y., Zhang Y., Huang C., Cui W. Biological and analytical variations of 16 parameters related to coagulation screening tests and the activity of coagulation factors // Seminars in Thrombosis and Hemostasis. 2015. vol. 41, № 3, pp. 336-341. doi:10.1055/s-0034-1543994

14. d'Audigier C., Delassasseigne C., Robert A., Eschwège V. (2016). Underestimation of plasma level of factor $\mathrm{V}$ coagulant activity and fibrinogen concentration together with prolonged prothrombin time, activated partial thromboplastin time and thrombin time can result from pre-analytical very low calcium level in citrated sample tube // International Journal of Laboratory Hematology. 2016. vol. 38, № 1. P. 50-53. doi:10.1111/ijlh.12434

15. Горбунова Е.В., Макаров С.А., Барбараш О.Л. Внедрение централизованного контроля МНО в Кузбассе // Комплексные проблемы сердечно-сосудистых заболеваний. 2016. Т. 5, № 2. С. 105-110.

16. Эффективность системы централизованного мониторинга МНО у пациентов, принимающих варфарин по поводу перенесенного венозного тромбоза / Хруслов М.В., Лобачев В.И., Уханова И.Ю., Егорчева Ю.С. // Флебология. 2013. T. 7, № 4. С. 52-56.

\section{References}

1. Berkovsky A.L., Sergeeva E.V., Prostakova T.M., Melkumyan A.L., Suvorov A. Skriningovye testy plazmennogo gemostaza protrombinovoe vremya, AChTV, trombinovoe vremya, fibrinogen [Screening tests of plasma hemostasis, Prothrombin time, APTT, Thrombin time, Fibrinogen]. Moscow, 2016, 70 p.

2. Titaeva E.V., Dobrovolsky A.B. Vozmozhnye istochniki oshibok pri opredelenii MNO i puti ikh resheniya [Challenges and possible solutions in determining INR] Atherothrombosis Journal [Aterotromboz], 2015, no. 2, pp. 107-114. doi:10.21518/2307-1109-2015-2-107-114

3. Guidelines for thromboplastin and plasma used to control oral anticoagulant therapy. WHO Expert Committee on Biological Standartization. Report 48. World Health Organization, 1999, pp. 64-69. (WHO technical report series; 889) 
4. Popov A., Mirkov I., Ninkov M., Mileusnic D., Demenesku J., Subota V., Kataranovski D., Kataranovski M. Effects of warfarin on biological processes other than haemostasis: A review. Food and Chemical Toxicology, 2018, vol. 113, pp. 19-32. doi.org/10.1016/j.fct.2018.01.019

5. Tan C.S.Y., Fong A.Y.Y., Jong Y.H., Ong T.K. INR Control of Patients with Mechanical Heart Valve on Long-Term Warfarin Therapy. Global Heart, 2018, vol. 13, no. 4, pp. 241-244. doi.org/10.1016/j.gheart.2018.08.003

6. Grzymala-Lubanski B., Svensson P.J., Renlund H., Jeppsson A., Själander A. Warfarin treatment quality and prognosis in patients with mechanical heart valve prosthesis. Heart, 2016, vol. 103, no. 3, pp. 198-203. doi:10.1136/heartjnl-2016-309585

7. Brasen C.L., Madsen J.S., Parkner T., Brandslund I. Home Management of Warfarin Treatment Through a Real-Time Supervised Telemedicine Solution: A Randomized Controlled Trial. Telemedicine and e-Health, 2018. doi:10.1089/ tmj.2017.0260

8. Smaradottir B., Martinez S., Borycki E., Loudon G., Kushniruk A., Jortveit J., Fensli R. User Evaluation of a Smartphone Application for Anticoagulation Therapy. Studies in Health Technology and Informatics, 2018, vol. 247, pp. 466-470. doi:10.3233/978-1-61499-852-5-466

9. Kirchhof P., Benussi S., Kotecha D., Ahlsson A., Atar D., Casadei B., Castella M., Diener H.C., Heidbuchel H., Hendriks J., Hindricks G., Manolis A.S., Oldgren J., Popescu B.A., Schotten U., Van Putte B., Vardas P. 2016 ESC Guidelines for the management of atrial fibrillation developed in collaboration with EACTS. European Heart Journal, 2016, vol. 37, no. 38, pp. 2893-2962. doi: 10.1093/eurheartj/ehw210

10. Bernaitis N., Badrick T., Davey A.K., Crilly J., Anoopkumar-Dukie S. Warfarin control in patients transitioning to warfarin after non-vitamin $\mathrm{K}$ oral anticoagulant (NOAC) therapy. Journal of Thrombosis and Thrombolysis, 2018, vol. 46, no. 4, pp. 461-465. doi: 10.1007/s11239-018-1719-x

11. Skaistis J., Tagami T. Risk of Fatal Bleeding in Episodes of Major Bleeding with New Oral Anticoagulants and Vitamin K Antagonists: A Systematic Review and Meta-Analysis. PLoS One, 2015, vol. 10, no. 9, pp. e0137444. doi: 10.1371/ journal.pone. 0137444

12. Banfi G., Del Fabbro M. (2008). Biological variation in tests of hemostasis. Seminars in Thrombosis and Hemostasis, 2008, vol. 34, no. 7, pp. 635-641. doi:10.1055/s-0028-1104541

13. Chen Q., Shou W., Wu W., Guo Y., Zhang Y., Huang C., Cui W. Biological and analytical variations of 16 parameters related to coagulation screening tests and 
the activity of coagulation factors. Seminars in Thrombosis and Hemostasis, 2015, vol. 41, no. 3, pp. 336-341. doi:10.1055/s-0034-1543994

14. d'Audigier C., Delassasseigne C., Robert A., Eschwège V. (2016). Underestimation of plasma level of factor $\mathrm{V}$ coagulant activity and fibrinogen concentration together with prolonged prothrombin time, activated partial thromboplastin time and thrombin time can result from pre-analytical very low calcium level in citrated sample tube. International Journal of Laboratory Hematology, 2016, vol. 38, no. 1, pp. 50-53. doi:10.1111/ijlh.12434

15. Gorbunova E.V., Makarov S.A., Barbarash O.L. Vnedrenie tsentralizovannogo kontrolya MNO v Kuzbasse [Introduction of centralized INR control in Kuzbass]. Kompleksnye problemy serdechno-sosudistykh zabolevaniy [Complex Issues of Cardiovascular Diseases], 2016, vol. 5, no. 2, pp. 105-110.

16. Khruslov M.V., Lobachev V.I., Ukhanova I.Iu., Egorcheva Iu.S. Effektivnost" sistemy tsentralizovannogo monitoringa mno u patsientov, prinimayushchikh varfarin po povodu perenesennogo venoznogo tromboza [The effectiveness of centralized monitoring the international normalized ratio in the patients treated with warfarin after deep venous thrombosis]. Flebologiya [Flebologiya], 2013, vol. 7 , no. 4 , pp. 52-56.

\section{ДАННЫЕ ОБ АВТОРАХ}

Аксютина Наталья Валерьевна, д.м.н., доцент кафедры внутренних болезней №1

ФГБОУ ВО КрасГМУ им. проф. В.Ф. Войно-Ясенецєкого Минздрава России

ул. Партизана Железняка, 1, г. Красноярск, 660022, Российская Федерачия

aks-n-v@yandex.ru

Шульман Владимир Абрамович, д.м.н., профессор кафедры внутренних болезней №1

ФГБОУ ВО КрасГМУ им. проф. В.Ф. Войно-Ясенецикого Минздрава России

ул. Партизана Железняка, 1, г. Красноярск, 660022, Российская Федерация

shulman36@mail.ru

Зограф Федор Георгиевич, к.т.Н., доцент кафедры приборостроения и наноэлектроники 
Сибирский федеральный университет

пр. Свободный, 79, г. Красноярск, 660041, Красноярский край, Российская Федераиия

fedor-zograf@yandex.ru

Мордовский Василий Сергеевич, врач-кардиолог, аспирант кафедры внутренних болезней №1

ФГБОУ ВО КрасГМУ им. проф. В.Ф. Войно-Ясенецкого Минздрава России

ул. Партизана Железняка, 1, г. Красноярск, 660022, Российская Федерачия vasek220@mail.ru

Маринушкин Павел Сергеевич, к.т.н., доцент кафедры приборостроения и наноэлектроники

Сибирский федеральный университет

пр. Свободныий, 79, г. Красноярск, 660041, Красноярский край, Российская Федерация

Гавриленко Михаил Игоревич, студент

Сибирский федеральный университет

пр. Свободный, 79, г. Красноярск, 660041, Красноярский край, Российская Федерачия

Кусаев Виктор Владимирович, к.м.н., доцент кафедры терапии ИПО ФГБОУ ВО КрасГМУ им. проф. В.Ф. Войно-Ясенецкого Минздрава России

ул. Партизана Железняка, 1, г. Красноярск, 660022, Российская Федерачия

vkusaev@gmail.com

Кононов Евгений Николаевич, врач-кардиолог, заместитель главного врача по клинико-экспертной работе

Краевое государственное бюджетное учреждение здравоохранения «Красноярская межрайонная клиническая больница № 20 имени И.С. Берзона»

ул. Инструментальная, 12, г. Красноярск, 660123, Российская Федерачия

e.kononov-76@mail.ru 
Петров Сергей Сергеевич, врач-кардиолог

Краевое государственное бюджетное учреждение здравоохранения «Краевая клиническая больница»

ул. Партизана Железняка, 1, г. Красноярск, 660022, Российская Федерачия

sergey_bayanist@mail.ru

Семёнов Эдуард Васильевич, к.м.н., ассистент кафедры онкологии и лучевой терапии с курсом ПО, врач-онколог

ФГБОУ ВО КрасГМУ им. проф. В.Ф. Войно-Ясенецкого Минздрава России

ул. Партизана Железняка, 1, г. Красноярск, 660022, Российская Федерачия

semenov_krasgmu@mail.ru

\section{Князева Ирина Сергеевна, студент}

ФГБОУ ВО КрасГМУ им. проф. В.Ф. Войно-Ясенецкого Минздрава России

ул. Партизана Железняка, 1, г. Красноярск, 660022, Российская Федерачия

vopros1211@yandex.ru

\section{DATA ABOUT THE AUTHORS}

Aksyutina Natalya Valeryevna, $\mathrm{PhD}$, MD, Associate Professor Department of Internal Diseases

Krasnoyarsk State Medical University by Prof. V.F. Voino-Yasenetsky

1, Partizan Zheleznyak Str., 660022, Krasnoyarsk, Russian Federation aks-n-v@yandex.ru

SPIN-code: 5028-3792

ORCID: 0000-0002-4856-2729

ResearcherID: F-3846-2016

Scopus Author ID: 55671852900

Shulman Vladimir Abramovich, $\mathrm{PhD}, \mathrm{ScD}$, Professor Department of internal Diseases

Krasnoyarsk State Medical University byProf. V.F. Voino-Yasenetsky 1, Partizan Zheleznyak Str., 660022, Krasnoyarsk, Russian Federation shulman36@mail.ru 
SPIN-code: $3845-0004$

Scopus Author ID: 7003452847

Zograf Fedor Georgievich, Candidate of Engineering Sciences, Department of Instrument Engineering and Nanoelectronics Siberian Federal University 79, Svobodny ave., 660041, Krasnoyarsk, Russian Federation fedor-zograf@yandex.ru Scopus Author ID: 56021779600 SPIN- code: $9079-4501$

Mordovsky Vasiliy Sergeyevich, MD, postgraduate student Krasnoyarsk State Medical University by Prof. V.F. Voino-Yasenetsky 1, Partizan Zheleznyak Str., 660022, Krasnoyarsk, Russian Federation vasek220@mail.ru SPIN-code: 7732-2117 ORCID: 0000-0001-9666-2487 ResearcherID: $K-7244-2018$ Scopus ID: 57194754937

Marinushkin Pavel Sergeevich, Candidate of Engineering Sciences, Department of Instrument Engineering and Nanoelectronics, Associate Professor

Siberian Federal University

79, Svobodny ave., 660041, Krasnoyarsk, Russian Federation

Scopus Author ID: 36628715400

ORCID: 0000-0001-7510-526X

SPIN-code: $5147-8169$

Gavrilenko Mikhail Igorevich, Student

Siberian Federal University

79, Svobodny ave., 660041, Krasnoyarsk, Russian Federation SPIN-code: 6394-3946

Kusaev Viktor Vladimirovich, $\mathrm{PhD}$, Associate Professor Krasnoyarsk State Medical University by Prof. V.F.Voino-Yasenetsky 1, Partizan Zheleznyak Str., 660022, Krasnoyarsk, Russian Federation vkusaev@gmail.com 
Kononov Evgeny Nikolayevich, MD, Deputy Chief Physician for Clinical and Expert Work

Krasnoyarsk Interdistrict Clinical Hospital № 20 by I.S. Berzona 12, Instrumentalnaya str., 660123, Krasnoyarsk, Russian Federation e.kononov-76@mail.ru

Petrov Sergey Sergeevich, MD, Regional Clinical Hospital, Cardiac Surgery Department

Krasnoyarsk State Medical University by Prof. V.F. Voino-Yasenetsky 1, Partizan Zheleznyak Str., 660022, Krasnoyarsk, Russian Federation sergey_bayanist@mail.ru

Semenov Eduard Vasilyevich, $\mathrm{PhD}$, MD, Assistant of the Department of Oncology Krasnoyarsk State Medical University by Prof. V.F. Voino-Yasenetsky

1, Partizan Zheleznyak Str., 660022, Krasnoyarsk, Russian Federation semenov_krasgmu@mail.ru SPIN-code: 5021-3613

Knyazeva Irina Sergeevna, Student

Krasnoyarsk State Medical University by Prof. V.F. Voino-Yasenetsky 1, Partizan Zheleznyak Str., 660022, Krasnoyarsk, Russian Federation vopros1211@yandex.ru

ORCID: 0000-0001-5147-5872

ResearcherID: $K-7042-2018$ 